\title{
Undersampled MR Image Reconstruction with Data-Driven Tight Frame
}

\author{
Jianbo Liu, ${ }^{1}$ Shanshan Wang, ${ }^{1,2}$ Xi Peng, ${ }^{1}$ and Dong Liang ${ }^{1}$ \\ ${ }^{1}$ Paul C. Lauterbur Research Centre for Biomedical Imaging, Shenzhen Institutes of Advanced Technology, \\ Chinese Academy of Sciences, Shenzhen 518055, China \\ ${ }^{2}$ School of Information Technologies, The University of Sydney, Sydney, NSW 2006, Australia
}

Correspondence should be addressed to Dong Liang; dong.liang@siat.ac.cn

Received 23 March 2015; Revised 10 June 2015; Accepted 10 June 2015

Academic Editor: Po-Hsiang Tsui

Copyright (C) 2015 Jianbo Liu et al. This is an open access article distributed under the Creative Commons Attribution License, which permits unrestricted use, distribution, and reproduction in any medium, provided the original work is properly cited.

\begin{abstract}
Undersampled magnetic resonance image reconstruction employing sparsity regularization has fascinated many researchers in recent years under the support of compressed sensing theory. Nevertheless, most existing sparsity-regularized reconstruction methods either lack adaptability to capture the structure information or suffer from high computational load. With the aim of further improving image reconstruction accuracy without introducing too much computation, this paper proposes a data-driven tight frame magnetic image reconstruction (DDTF-MRI) method. By taking advantage of the efficiency and effectiveness of datadriven tight frame, DDTF-MRI trains an adaptive tight frame to sparsify the to-be-reconstructed MR image. Furthermore, a twolevel Bregman iteration algorithm has been developed to solve the proposed model. The proposed method has been compared to two state-of-the-art methods on four datasets and encouraging performances have been achieved by DDTF-MRI.
\end{abstract}

\section{Introduction}

Magnetic Resonance Imaging (MRI) is one of the major diagnostic imaging modalities with noninvasive and nonionizing radiation nature. However, relatively low imaging speed limits its wide application in clinic [1]. To accelerate MRI, one popular way is to reduce the number of acquired data [2]. With $f \in \mathbb{C}^{m}$ and $x \in \mathbb{C}^{n}$, respectively, denoting the $K$-space measurement and the original image, the imaging model could be described as follows:

$$
f=P F x+\epsilon=F_{p} x+\epsilon,
$$

where $\epsilon$ is the complex additive white Gaussian noise with standard deviation $\sigma, P$ is the undersampling operator, $F$ is the Fourier operator, and $F_{p}=P F$.

Recovering $x$ from the undersampled measurement $f$ is an ill-posed inverse problem. To address this ill-posed nature, it is necessary to utilize some prior knowledge to regularize the MR image so as to make up the missing information. With the popularity of compressed sensing (CS) theory $[3,4]$, the sparsity-promoting regularization for MR image reconstruction has attracted many researchers $[2,5$, 6]. Specifically, the CS theory has shown that if an image has a sparse representation under certain transform, we can precisely restore the original image from its partial measurements under the RIP condition $[3,4]$. With such a transform, the MR image reconstruction from its undersampled $K$ space data can be achieved by nonlinear algorithms, like $\ell_{1}$ minimization or orthogonal match pursuit (OMP) algorithm $[2,5,6]$.

The commonly used transforms in MR image reconstruction include total variation (TV) and wavelet transform, both of which can be regarded as tight frames [7-13]. As the tight frame satisfies the perfect reconstruction property, which ensures that the given signal can be perfectly represented by its canonical representation, it has been leveraged in diverse inverse problems $[7,11,12]$. However, TV prior assumes that the image consists of piecewise constant areas which may not be valid in many real MR images. When the measurements are highly undersampled, compressed sensing based MRI using TV regularization (CSMRI-TV) could lead to severe blocky artifacts $[2,14]$. To improve the image quality, 
Liang et al. [15] applied the nonlocal total variation (NLTV) regularization in parallel MR imaging by replacing the gradient functional in conventional TV with a weighted nonlocal gradient function. However, although NLTV reduces the blocky effects, it is still based on fixed transform, which does not adapt to the target image. Furthermore, other analytically designed X-lets $[8-10,16]$, such as wavelets and shearlets, also suffer from their intrinsic deficiencies [17], such as the dependency between parent and child wavelet coefficients and the lack of adaptability. Qu et al. [18] introduced the patch-based directional wavelet (PBDW) in MR image reconstruction by exploiting the geometric direction of image patches, which has shown encouraging performances on edge preservation and noise removal. Nevertheless, PBDW is still a simplified form of bandlets and the adaptability can still be explored [19].

Since the fixed tight frame/transform might not be universally optimal for all images, the data-driven tight frame/transform has been developed $[5,6,20,21]$ to adaptively capture the structure information. One most popular direction is the incorporation of the dictionary learning (DL) into MRI [6, 21, 22], among which Ravishankar and Bresler proposed a benchmark work named DLMRI [5] with outstanding reconstruction results achieved. However, dictionary learning based optimization is a large-scale and highly nonconvex problem, which requires high computational complexity [6, 20]. More recently, Cai et al. [20] presented a scheme to learn a data-driven tight frame from the measurements and applied it to solve the image denoising problem. As the data-driven tight frame satisfies the perfect reconstruction property, it is very efficient to obtain the results with less artifacts. Therefore, Wang et al. [23] tried to incorporate data-driven tight frame into dynamic MRI. However, the proposed method relies on a perfect reference image, which is quite hard to be obtained from its undersampled $K$ space data, and the data-driven tight frame is learnt from the reference image instead of the target image.

Based on these observations and motivated by the efficiency and effectiveness of the data-driven tight frame (DDTF), in this work, we propose an undersampled MR imaging method by incorporating DDTF into the reconstruction model with the aim of further improving the image reconstruction accuracy without introducing too much computation. Specifically, a tight frame has been adaptively trained for each to-be-reconstructed MR image. To solve the proposed model, a two-level Bregman iteration algorithm has been developed. We name the proposed approach as DDTFMRI and compare it to two state-of-the-art MR image reconstruction algorithms, including CSMRI-TV and DLMRI, on one simulated MR image and three in vivo datasets under different undersampling scenarios. The experiments have shown encouraging performances of the proposed method.

The rest of the paper is organized as follows. Section 2 presents the preliminary and previous work. Our proposed method DDTF-MRI is illustrated in Section 3. The experimental results are provided in Section 4 demonstrating that the proposed algorithm has potential to improve the MR image reconstruction from its undersampled $K$-space data. Finally, conclusions are given in Section 5.

\section{Preliminary and Previous Work}

2.1. Tight Frame. A system $\left\{x_{i}\right\} \subset L_{2}(\mathbb{C})$ is a tight frame $[7$, 24] of $L_{2}(\mathbb{C})$ if

$$
g=\sum_{i}\left\langle g, x_{i}\right\rangle x_{i}, \quad \forall g \in L_{2}(\mathbb{C}),
$$

where $\langle\cdot, \cdot\rangle$ is the inner product operator and the space $L_{2}(\mathbb{C})$ represents the set of all the functions $f(\theta)$ satisfying $\|f\|_{L_{2}(\mathbb{C})}:=\left(\int_{\mathbb{C}}|f(\theta)| d \theta\right)^{1 / 2}$. For a given tight frame, we can define the corresponding analysis operator $W$ as

$$
W: g \in L_{2}(\mathbb{C}) \longrightarrow\left\{\left\langle x, x_{i}\right\rangle\right\} \in \ell_{2}(\mathbb{N})
$$

and the synthesis operator $W^{T}$ as

$$
W^{T}:\left\{c_{i}\right\} \in \ell_{2}(\mathbb{N}) \longrightarrow \sum_{n} c_{i} x_{i} \in L_{2}(\mathbb{C}) .
$$

The system is a tight frame if and only if $W^{T} W=I$, where $I$ is the identity operator. Moreover, a tight frame can be generated from a filter bank satisfying the Unitary Extension Principle (UEP) condition [7]. Let $\left\{a_{i}\right\}_{i=1}^{i=r^{2}}$ denote the set of 2D filters and the size of $a_{i}$ is $r \times r$ and then the synthesis operator $W^{T} \in \mathbb{C}^{n \times n r^{2}}$ and the analysis operator $W \in \mathbb{C}^{n r^{2} \times n}$ are defined as

$$
\begin{aligned}
W^{T} & =\left[S_{a_{1}}, S_{a_{2}}, \ldots, S_{a_{r^{2}}}\right], \\
W & =\left[S_{a_{1}}^{T}, S_{a_{2}}^{T}, \ldots, S_{a_{r^{2}}}^{T}\right]^{T},
\end{aligned}
$$

where $S_{a_{i}} \in \mathbb{C}^{n \times n}$ denotes the convolution matrix associated with the filter $a_{i}$, which is a block-wise Toeplitz matrix under Neumann boundary conditions [7, 24].

2.2. Sparsity-Regularized MR Image Reconstruction. This subsection briefly introduces two representative sparsityregularized MR image reconstruction methods, namely, CSMRI and DLMRI. CSMRI is a classical approach exploiting sparsity via fixed transform and its mathematical model can be defined as follows:

$$
\begin{array}{ll}
\min _{x} & \|W x\|_{1}, \\
\text { s.t. } & \left\|f-F_{p} x\right\|^{2} \leq \sigma^{2},
\end{array}
$$

where $W$ is the analysis operator of its corresponding frame system, that is, wavelet or total variation.

DLMRI proposed by Ravishankar and Bresler [5], on the other hand, employs the $K$-singular value decomposition (K-SVD) algorithm [21] to learn an adaptive dictionary to sparsely represent the patches extracted from the target image. The DLMRI model can be written as

$$
\begin{array}{ll}
\min _{x, D, \Gamma} & \sum_{i}\left\|R_{i} x-D \alpha_{i}\right\|_{2}^{2}+\lambda \sum_{i}\left\|\alpha_{i}\right\|_{0}, \\
\text { s.t. } & \left\|f-F_{p} x\right\|^{2} \leq \sigma^{2},
\end{array}
$$


where $D$ represents the dictionary, $R_{i}$ denotes the operator that extracts the $i$ th patch from the image $x$, and $\Gamma$ denotes the set of all sparse coefficients $\left\{\alpha_{i}\right\}$.

\section{The Proposed DDTF-MRI Method}

3.1. Image Reconstruction Model. To enhance the sparsity in the frame domain, the proposed DDTF-MRI method is devoted to learning an adaptive data-driven tight frame to effectively sparsify the target image. The proposed model can be described as

$$
\begin{aligned}
\min _{x, W \in \Lambda} & \|W x\|_{1}, \\
\text { s.t. } & \left\|f-F_{p} x\right\|^{2} \leq \sigma^{2},
\end{aligned}
$$

where $\Lambda=\left\{W \mid W^{T} W=I\right\}$ and the tight frame $W$ is constructed by the corresponding 2D filters $\left\{a_{i}\right\}_{i=1}^{i=r^{2}}$ in the manner of (5).

3.2. The Proposed Algorithm. A two-level Bregman iteration algorithm has been developed to attack the target model.

3.2.1. The Outer Bregman Iteration. In the outer-level Bregman iteration, we try to solve the following problem $[6,25$, 26]:

$$
\begin{aligned}
\left\{x^{k+1}, W^{k+1}\right\} & =\underset{x, W \in \Lambda}{\operatorname{argmin}}\|W x\|_{1}+\frac{\mu}{2}\left\|F_{p} x-f+c^{k}\right\|_{2}^{2}, \\
c^{k+1} & =c^{k}+\delta_{c}\left(F_{p} x^{k+1}-f\right),
\end{aligned}
$$

where $\mu>0$ and $\delta_{c} \in(0,2)[26]$.

3.2.2. The Inner Bregman Iteration. With introduction of an assistant variable, $v=W x$, we can obtain the constrained version of the first subproblem in (9):

$$
\begin{aligned}
\min _{x, W \in \Lambda, v} & \|v\|_{1}+\frac{\mu}{2}\left\|F_{p} x-f+c^{k}\right\|_{2}^{2}, \\
\text { s.t. } & v=W x .
\end{aligned}
$$

Employing the Bregman iteration technique again under the assistance of a dual variable, $b$, in the inner-level Bregman iteration, we target solving

$$
\begin{aligned}
x^{k+1}= & \underset{x}{\operatorname{argmin}} \frac{\mu}{2}\left\|F_{p} x-f+c^{k}\right\|_{2}^{2} \\
& +\frac{\lambda}{2}\left\|W x-v^{k}+b^{k}\right\|_{2}^{2}, \\
\left\{v^{k+1}, W^{k+1}\right\}= & \underset{v, W \in \Lambda}{\operatorname{argmin}}\|v\|_{1}+\frac{\lambda}{2}\left\|W x^{k+1}-v+b^{k}\right\|_{2}^{2}, \\
b^{k+1}= & b^{k}+\delta_{b}\left(W^{k+1} x^{k+1}-v^{k+1}\right),
\end{aligned}
$$

where $\lambda>0$ and $0<\delta_{b} \leq 1[26]$.
3.2.3. Subproblems of (11). For the $x$-subproblem in (11), as $W^{T} W=I$, its least squares solution can be obtained as

$$
\left(\mu F_{p}^{T} F_{p}+\lambda I\right) x^{k+1}=\mu F_{p}^{T}\left(f-c^{k}\right)+\lambda W^{T}\left(v^{k}-b^{k}\right) .
$$

Multiplying both sides of (12) by $F$ and letting $S_{1}=f-$ $c^{k}$ and $S_{2}=F\left[W^{T}\left(v^{k}-b^{k}\right)\right]$, we obtain the interpolation formulation in the frequency domain:

$$
\begin{aligned}
& F x^{k+1}\left(k_{x}, k_{y}\right) \\
& \quad= \begin{cases}S_{2}\left(k_{x}, k_{y}\right), & \left(k_{x}, k_{y}\right) \notin \Omega, \\
\frac{\mu S_{1}\left(k_{x}, k_{y}\right)+\lambda S_{2}\left(k_{x}, k_{y}\right)}{\mu+\lambda}, & \left(k_{x}, k_{y}\right) \in \Omega,\end{cases}
\end{aligned}
$$

where $\Omega$ stands for the subset of $K$-space that has been sampled. The image can then be updated via the inverse Fourier transform.

Next, we apply the alternating-minimization strategy to solve the $\{v, W\}$-subproblems:

$$
\min _{v, W \in \Lambda}\|v\|_{1}+\frac{\lambda}{2}\left\|W x^{k+1}-v+b^{k}\right\|_{2}^{2} .
$$

With $W$ temporarily fixed, the $v$-subproblem becomes

$$
v^{k+1 / 2}=\underset{v}{\operatorname{argmin}}\|v\|_{1}+\frac{\lambda}{2}\left\|W x^{k+1}-v+b^{k}\right\|_{2}^{2} .
$$

Following the iterative shrinkage/thresholding algorithm (ISTA) [27], it yields

$$
v^{k+1 / 2}=\operatorname{shrink}\left(W x^{k+1}+b^{k}, \frac{1}{\lambda}\right),
$$

where $\operatorname{shrink}(x, a)=\operatorname{sign}(x) \max \{0,|x|-a\}$. Now let $v$ be kept fixed; the $W$-subproblem turns to be

$$
W^{k+1}=\underset{W \in \Lambda}{\operatorname{argmin}}\left\|W x^{k+1}-v^{k+1 / 2}+b^{k}\right\|_{2}^{2} .
$$

Instead of directly optimizing $W$, we apply the technique of [20] to solve this subproblem by using SVD to obtain the corresponding filters $\left\{a_{i}\right\}$.

Furthermore, for a better restoration result, we also update the variable $v$ in the updated tight frame domain

$$
v^{k+1}=\underset{v}{\operatorname{argmin}}\|v\|_{1}+\frac{\lambda}{2}\left\|W^{k+1} x^{k+1}-v+b^{k}\right\|_{2}^{2}
$$

and obtain

$$
v^{k+1}=\operatorname{shrink}\left(W^{k+1} x^{k+1}+b^{k}, \frac{1}{\lambda}\right) .
$$

To help the readers grasp the overall picture better, we summarize the entire process in Algorithm 1. 


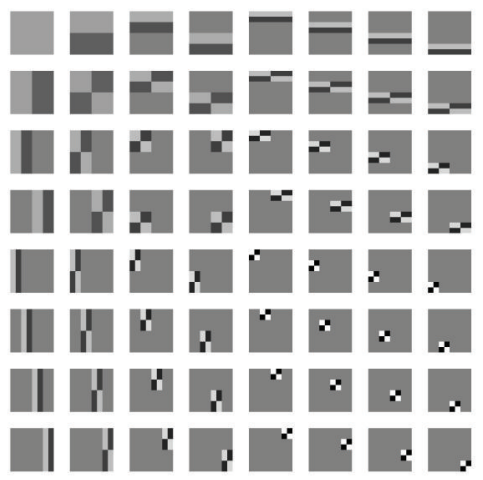

(a)

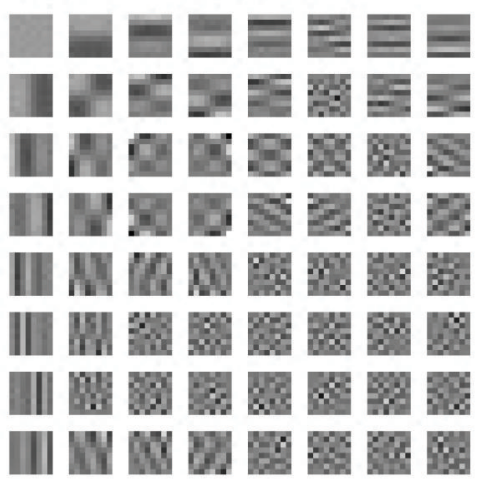

(b)

FIGURE 1: (a) Original filters. (b) The corresponding learnt filters in the experiment of Figure 2.

\author{
(1) Initialization: $v^{0}=0, b^{0}=0, c^{0}=0, W^{0}, k=0$ \\ (2) while stop condition is not met do \\ (3) for $m=1$ to $M$ do \\ (4) update $x^{k+1}$ according (13) \\ (5) update $v^{k+1 / 2}$ according (16) \\ (6) update $W^{k+1}$ according (17) \\ (7) update $v^{k+1}$ according (19) \\ (8) $b^{k+1}=b^{k}+\delta_{b}\left(W^{k+1} x^{k+1}-v^{k+1}\right)$ \\ (9) end for \\ (10) $c^{k+1}=c^{k}+\delta_{c}\left(F_{p} x^{x+1}-f\right)$ \\ (11) $k \leftarrow k+1$ \\ (12) end while
}

Algorithm 1: DDTF-MRI.

\section{Results and Discussion}

4.1. Experimental Setup. We evaluated the proposed DDTFMRI on four datasets and compared it to two state-of-theart methods, including CSMRI-TV (the CSMRI-TV code is available in http://www.eecs.berkeley.edu/ mlustig/CS.html) and DLMRI (the DLMRI code is available in http://www .ifp.illinois.edu/ yoram/DLMRI-Lab/DLMRI.html) [5]. The four datasets consist of one simulated data and three in vivo datasets. The simulated data is from the paper in [5]. For the in vivo data, informed consent was obtained from the volunteer in accordance with the institutional review board policy. The first in vivo data is a sagittal brain collected on a GE 3T scanner (GE Healthcare, Waukesha, WI, USA) with 32-channel head coil and 3D T1-weighted spoiled gradient echo sequence: $\mathrm{TE}=$ minimum full, $\mathrm{TR}=7.5 \mathrm{~ms}, \mathrm{FOV}=$ $24 \times 24 \mathrm{~cm}$, matrix $=256 \times 256$, and slice thickness $=1.7 \mathrm{~mm}$. The heart dataset was acquired on a $1.5 \mathrm{~T}$ Philips scanner using the steady-state free precession (SSFP) sequence with a flip angle of 50 degree and $\mathrm{TR}=3.45 \mathrm{msec}$. The field of view $(\mathrm{FOV})$ was $345 \mathrm{~mm} \times 270 \mathrm{~mm}$ and the slice thickness was $10 \mathrm{~mm}$. Retrospective cardiac gating was used with a heart rate of $66 \mathrm{bpm}$. The third in vivo data was acquired on a $3 \mathrm{~T}$ commercial scanner (GE Healthcare, Waukesha,
WI, USA) and eight-channel head coil (Invivo, Gainesville, FL, USA) with a 2D T1-weighted spin-echo protocol (axial plane, TE/TR $=11 / 700 \mathrm{~ms}, 22 \mathrm{~cm}$ FOV, 10 slices, matrix size $=256 \times 256)$. The adaptive combination method [28] is applied to integrate the multichannel data into a singlechannel complex-valued image before we apply the three methods. Then the full $K$-space data corresponding to the single channel image is retrospectively undersampled under different sampling schemes.

As shown in Figure 1(a), we employed the 3-level shiftinvariant Haar wavelet filters (the size of each filter is $8 \times 8$ ) as the initialization of the tight frame in DDTF-MRI. As for the parameter settings, both CSMRI-TV and DLMRI were implemented with their default settings. For DDTF-MRI, we set $M=3, \delta_{b}=1, \delta_{c}=1, \mu=10$, and $\lambda=10$. The outer-level iteration of DDTF-MRI continues until $k>25$. Furthermore, we used both the peak signal-to-noise ratio (PSNR) (the PSNR is defined as PSNR $=20 \log _{10} 255 /$ RMSE, where the RMSE is the root mean error estimated between the ground truth and the reconstructed image), high-frequency error norm (HFEN) [5], and structural similarity (SSIM) index [29] for a quantitative comparison of recovered results.

4.2. Test on the Simulated Data. We firstly applied each algorithm to reconstruct the simulated T2-weighted image under the 1D Cartesian sampling schemes with accelerating factor $R=6.7$ (sampling ratio 15\%). The filters learned by DDTF-MRI are shown in Figure 1(b). Compared with the initialization filter presented in Figure 1(a), we can see that the learnt filters have captured more directional information. Figures 2(a) and 2(b), respectively, present the original image of size $512 \times 512$ and the sampling mask. The evolution of the PSNR, HFEN, and SSIM values over iteration number is plotted in Figures 2(c), 2(d), and 2(e), which indicates that our proposed method is superior to the other two algorithms. The final PSNR values obtained by CSMRI-TV, DLMRI, and DDTF-MRI are, respectively, $32.97 \mathrm{~dB}, 35.34 \mathrm{~dB}$, and $38.03 \mathrm{~dB}$. Figures $2(\mathrm{f}), 2(\mathrm{~g})$, and $2(\mathrm{~h})$ provide the absolute reconstruction differences between the original image and the results obtained by the three methods, from which we can see the proposed method produces less estimation error. 


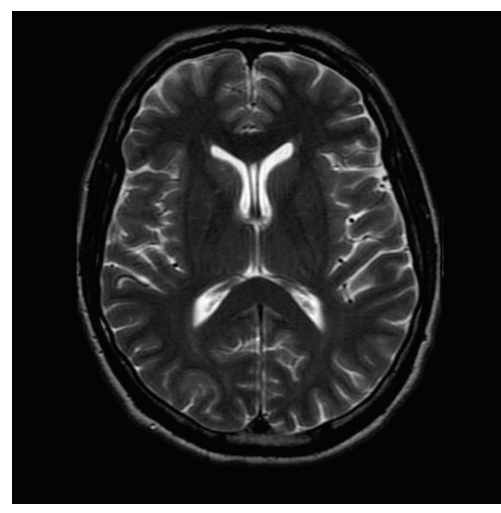

(a)

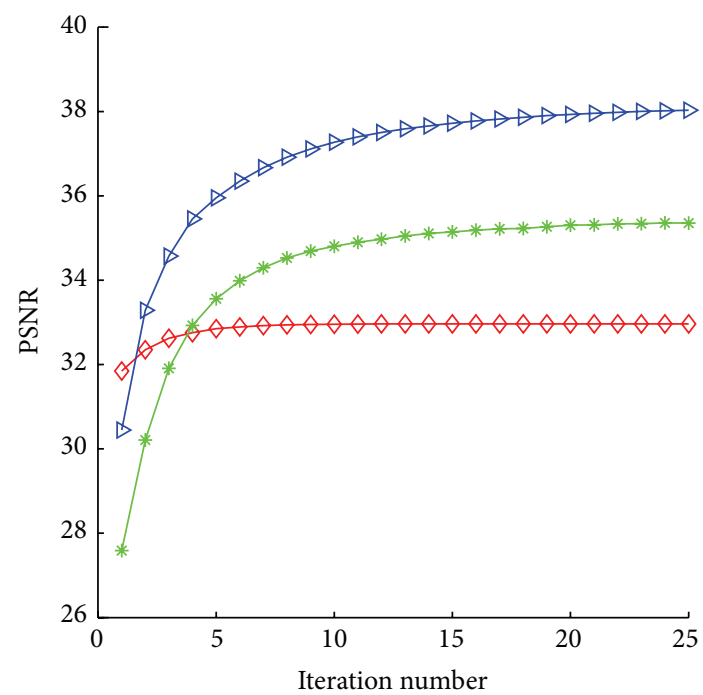

$\rightarrow$ CSMRI-TV
$\rightarrow$ DLMRI

$\rightarrow$ DDTF-MRI

(c)

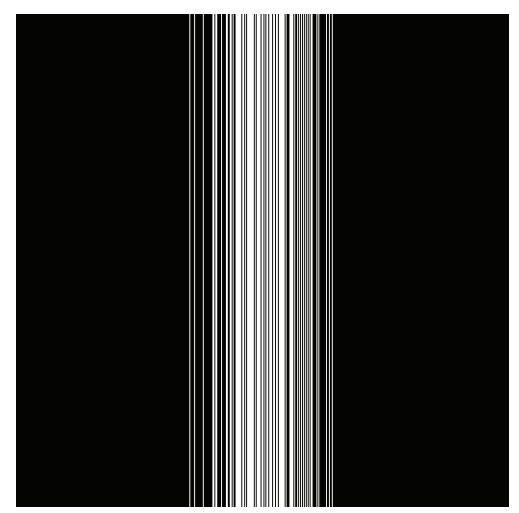

(b)

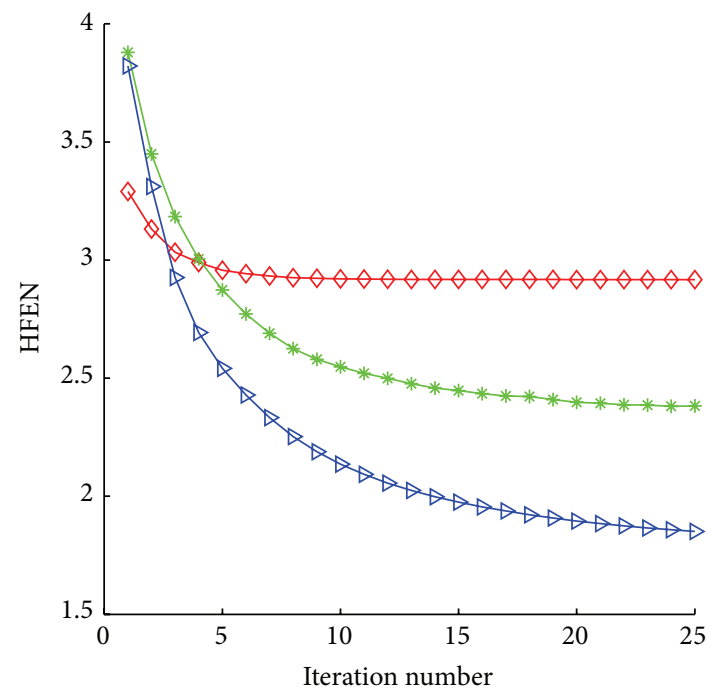

$\rightarrow$ CSMRI-TV

- DLMRI

$\rightarrow$ DDTF-MRI

(d)

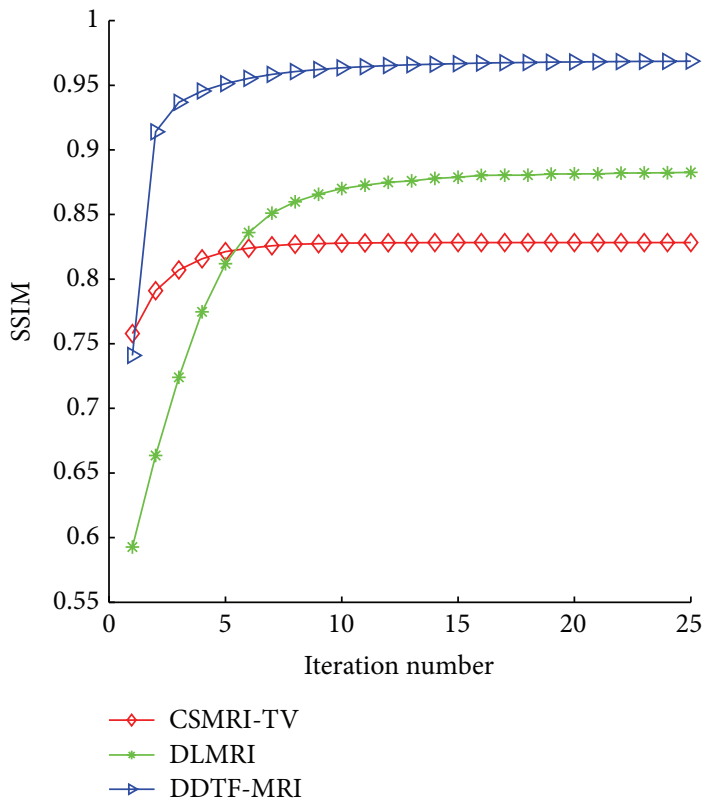

(e)

FIgUre 2: Continued. 


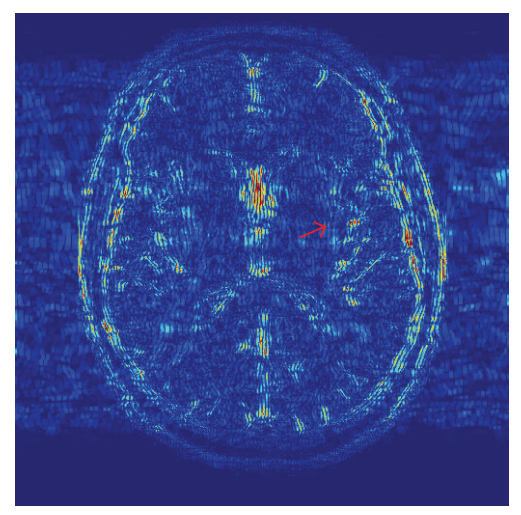

(f)

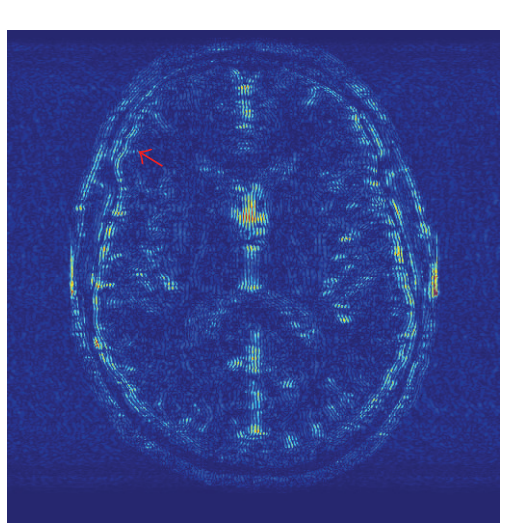

(g)

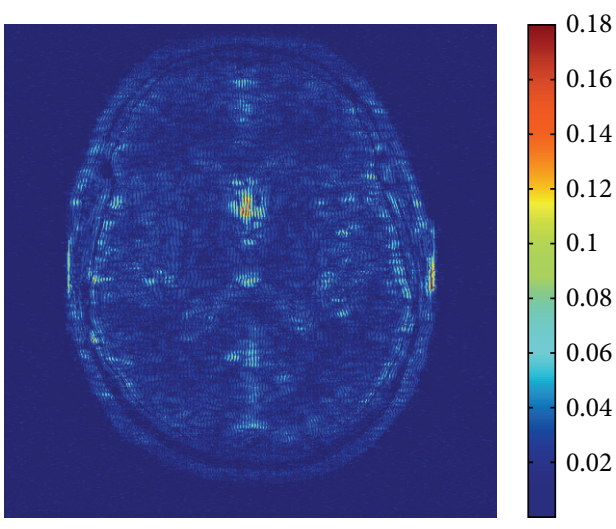

(h)

Figure 2: (a) Original image. (b) Cartesian sampling mask with accelerating factor $R=6.7$. (c), (d), and (e) are the PSNR, HFEN, and SSIM values versus the number of iterations. (f), (g), and (h) are reconstruction error magnitudes for CSMRI-TV, DLMRI, and DDTF-MRI, respectively.

TABLE 1: ROI analysis of mean and standard deviation (std).

\begin{tabular}{lccc}
\hline Image & \multicolumn{3}{c}{ Different ROIs } \\
& $\begin{array}{c}\text { ROI 1 } \\
\text { Mean } \pm \text { Std }\end{array}$ & $\begin{array}{c}\text { Mean } \pm \text { Std } \\
\text { ROI 3 } \\
\text { Mean } \pm \text { Std }\end{array}$ \\
\hline Reference & $35.34 \pm 5.55$ & $29.72 \pm 2.97$ & $39.08 \pm 5.87$ \\
CSMRI-TV & $35.47 \pm 7.31$ & $29.69 \pm 3.70$ & $39.01 \pm 7.89$ \\
DLMRI & $35.14 \pm 4.77$ & $29.40 \pm 2.04$ & $38.83 \pm 5.19$ \\
DDTF-MRI & $35.33 \pm 5.35$ & $29.75 \pm 2.67$ & $39.09 \pm 5.86$ \\
\hline
\end{tabular}

Moreover, a region-of-interest (ROI) analysis was performed by calculating means and standard deviations in selected ROIs. Table 1 shows the numerical result calculated from three selected ROIs in this experiment. The numbers of pixels in ROI 1, ROI 2, and ROI 3 are 1944, 1248, and 1944, respectively. From Table 1, we can find the values of our reconstruction result are closest to the values of reference image, which illustrates that our method can give a more precise reconstruction result.

4.3. Test on In Vivo Datasets. We then employed these three methods to reconstruct the sagittal brain and test their sensitivity to the acceleration factors. Figure 3(c) plots the PSNR values versus different accelerating factors under the random sampling trajectory. It shows that DDTF-MRI performs better than the other two methods at all the acceleration factors. For a visual comparison, Figure 4 provides the reconstructed results at accelerating factor 3 . For a close-up comparison, we have enlarged two edge parts, from which we can see DDTF-MRI provides clearer details.

To investigate the sensitivity of various methods to noise, CSMRI-TV, DLMRI, and DDTF-MRI were applied to reconstruct the heart image under pseudoradial sampling scheme with accelerating factor $R=3$. Figure 5(c) provides the PSNR values of the MR images reconstructed by CSMRITV (red curves), DLMRI (green curves), and DDTF-MRI (blue curves) versus diverse noise levels $(\sigma=2,4,6,8)$.
TABLE 2: Computational time (s) of different algorithms.

\begin{tabular}{lccc}
\hline \multirow{2}{*}{ Image } & \multicolumn{3}{c}{ Different algorithms } \\
& CSMRI-TV & DLMRI & DDTF-MRI \\
\hline Figure 2(a) & 147.3 & 1600.6 & 310.2 \\
Figure 4(a) & 30.9 & 2609.2 & 314.5 \\
Figure 5(a) & 37.1 & 2484.3 & 315.3 \\
Figure 6(a) & 31.5 & 2503.6 & 313.7 \\
\hline
\end{tabular}

We can see that our proposed DDTF-MRI exhibits the best performance for these noise levels. Figures 5(d), 5(e), and 5(f) show the reconstructed error magnitudes from the 3 -fold radial undersampled data with noise standard deviation as 4 . We can find that our method can preserve more detail and recover the structures more precisely.

In Figure 6, we evaluated the proposed method with another in vivo brain data, which contains more fine-detailed structures, using the radial sampling trajectory. The reconstructed results using CSMRI-TV, DLMRI, and DDTF-MRI with a higher acceleration factor $R=4$ are displayed in Figures 6(b), 6(c), and 6(d), respectively. The zoom-in results are also provided in Figure 6. Compared to the reference image shown in Figure 6(a), it can be observed that the result obtained by CSMRI-TV suffers from blocky artifacts. Meanwhile, the reconstructed image obtained by DDTF-MRI is clearer and sharper than those reconstructed by CSMRITV and DLMRI. This reveals that our proposed method can provide a more accurate recovered image.

4.4. Computation Time. In our experiments, all algorithms were implemented in MATLAB and performed on a laptop equipped with Intel $2.60 \mathrm{GHz}$ CPU and 4 GB RAM. Table 2 illustrates the cost time for these experiments. These computational times were obtained by averaging 10 times for each experiment. The size of the simulated image (Figure 2(a)) is $512 \times 512$ and the size of complex-valued images (Figures 4(a), 5(a), and 6(a)) is $256 \times 256$. As CSMRI-TV is based 

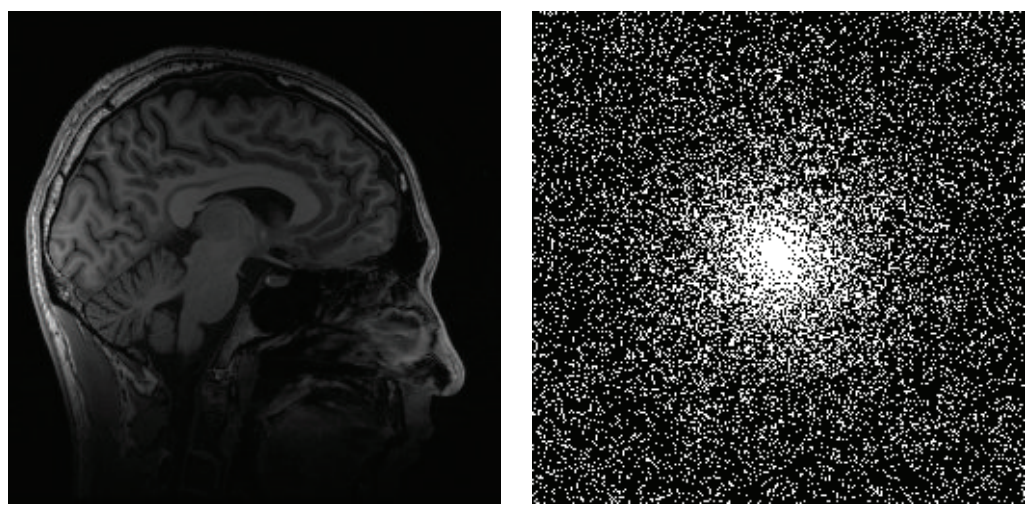

(a)

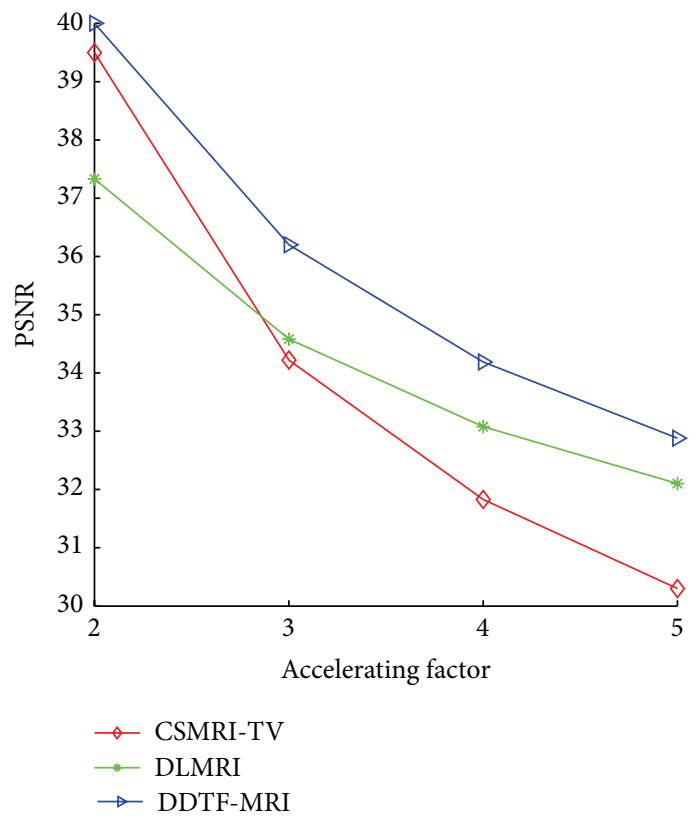

(c)

FIgURE 3: (a) Original image. (b) Random sampling mask. (c) PSNR values versus accelerating factors.

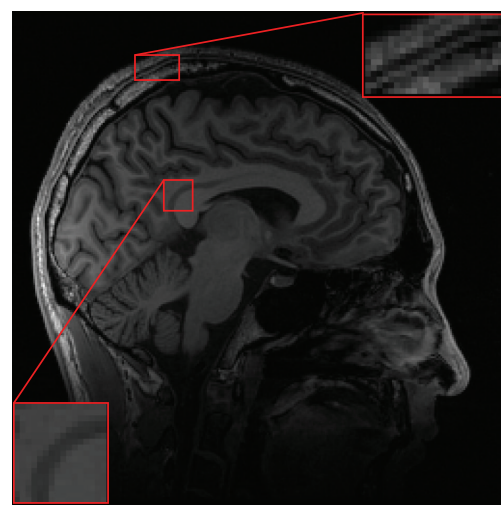

(a)

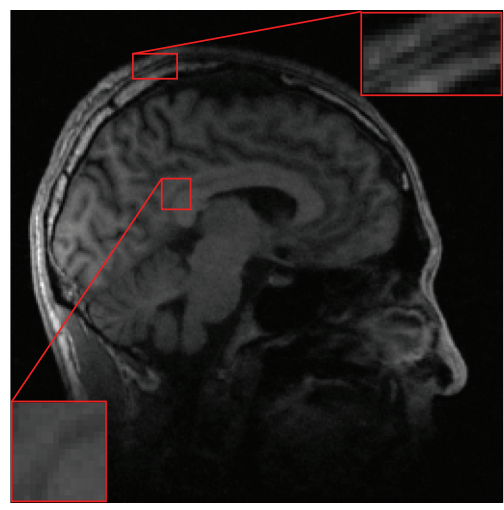

(c)

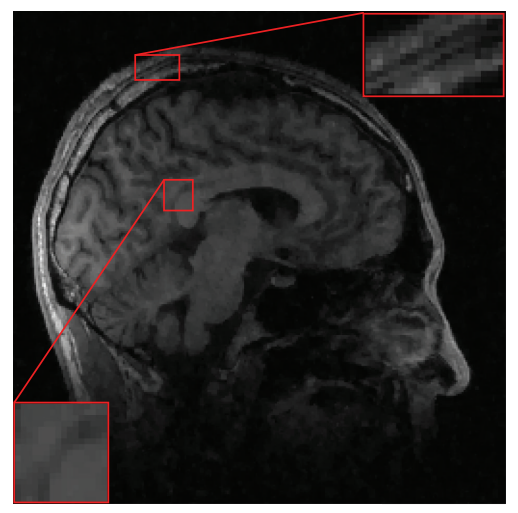

(b)

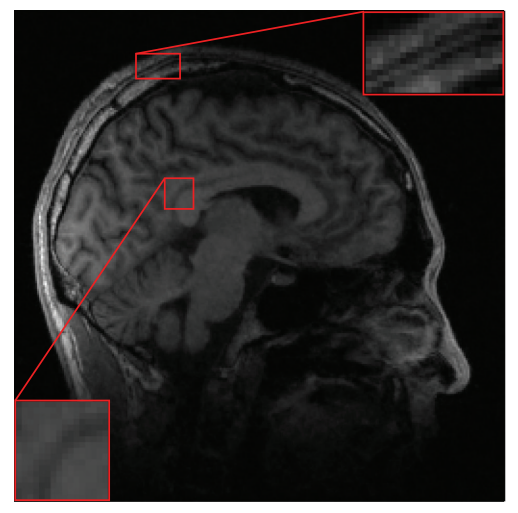

(d)

FIgURE 4: (a) Reference. Reconstructed images from the random sampling mask with accelerating factor $R=3$ by (b) CSMRI-TV, (c) DLMRI, and (d) our proposed DDTF-MRI, respectively. 


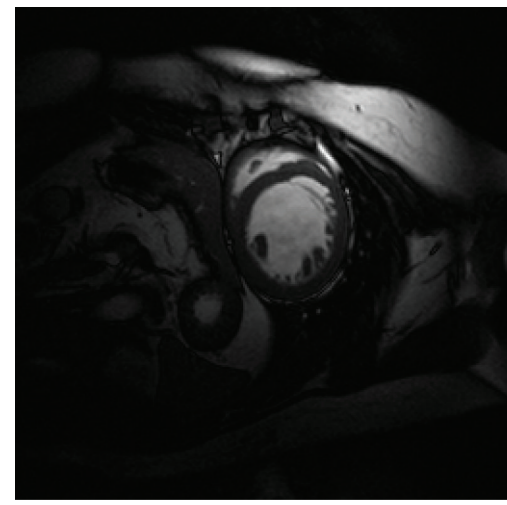

(a)

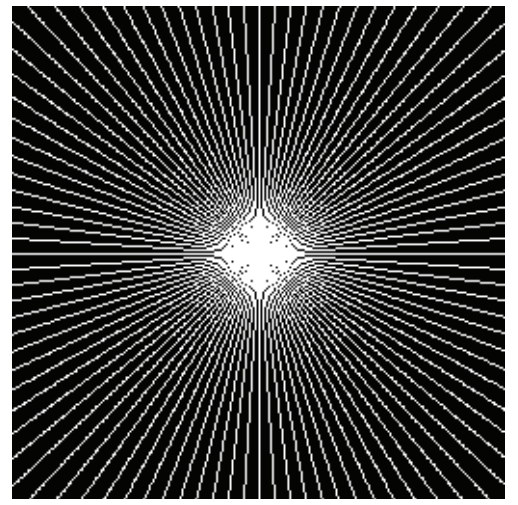

(b)

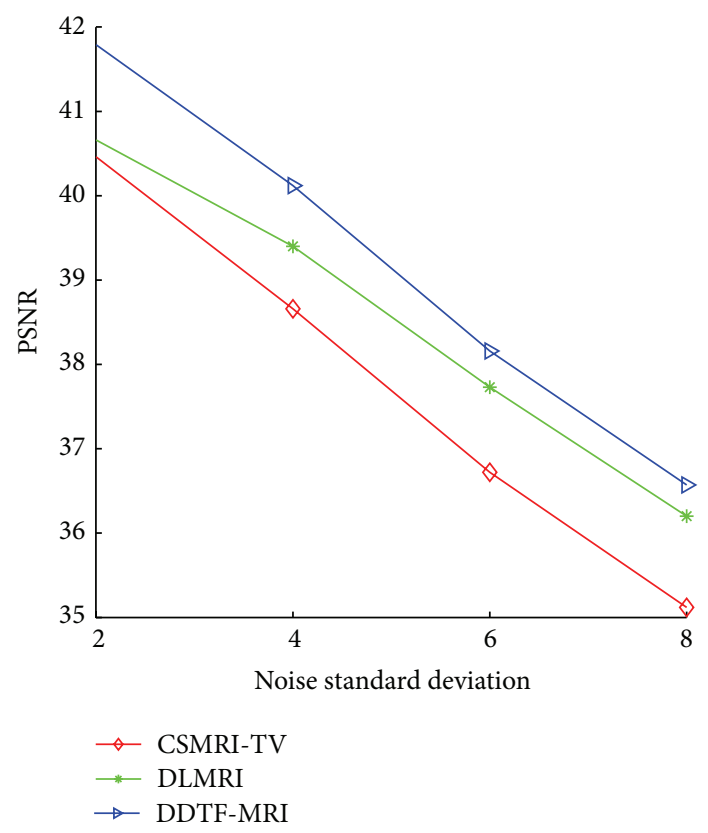

(c)

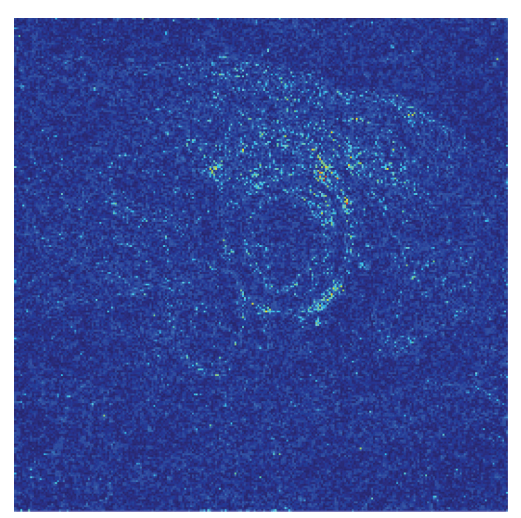

(d)

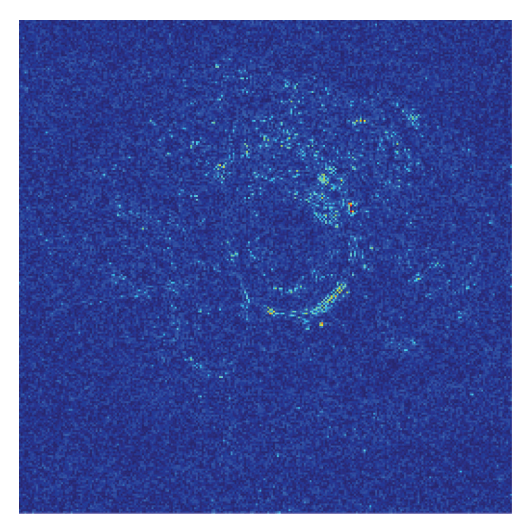

(e)

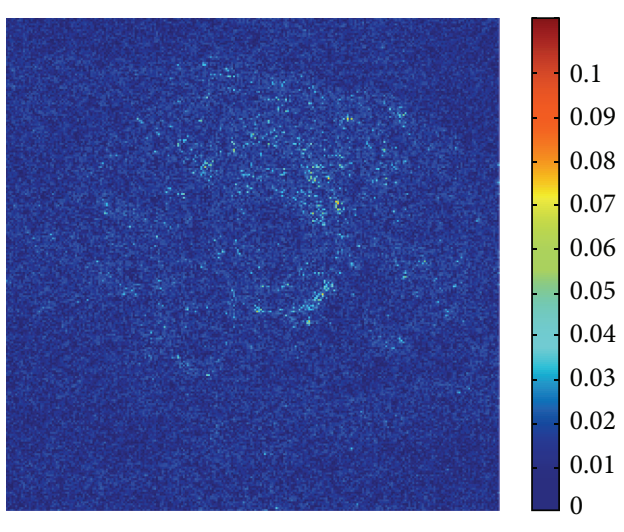

(f)

FIgURE 5: (a) Original image. (b) Radial sampling mask. (c) PSNR versus noise standard deviation. The error magnitudes of reconstructed results from the 3 -fold radial sampling mask with noise standard deviation 4 by (d) CSMRI-TV, (e) DLMRI, and (f) our proposed DDTF, respectively. 


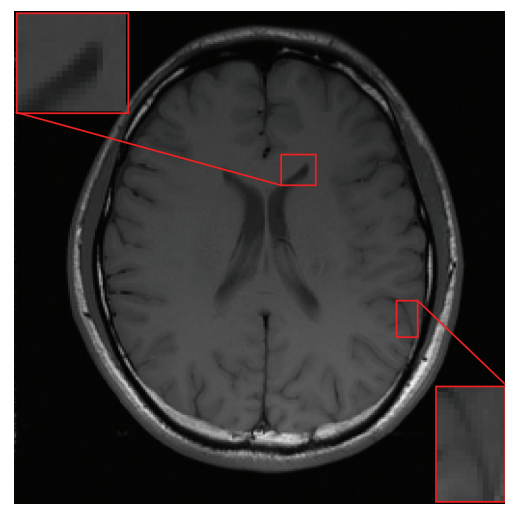

(a)

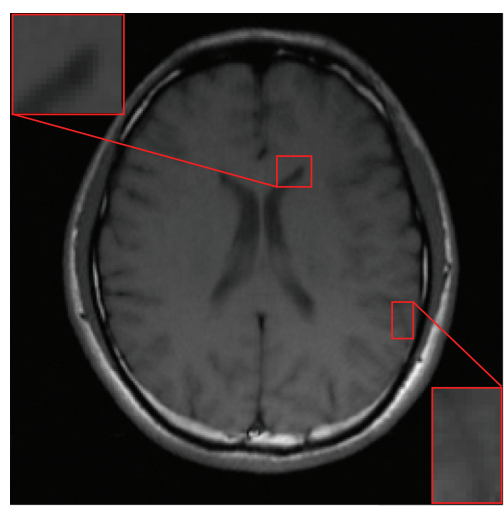

(c)

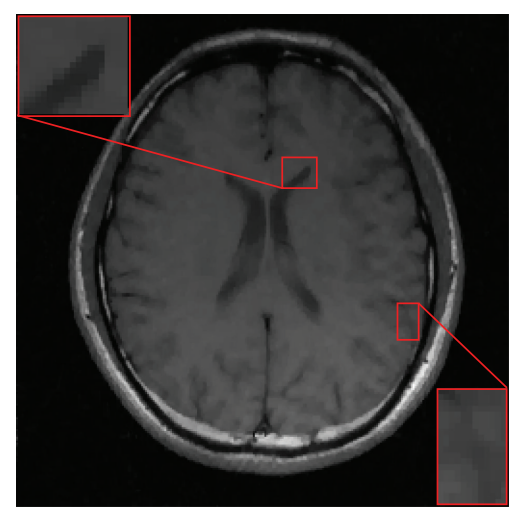

(b)

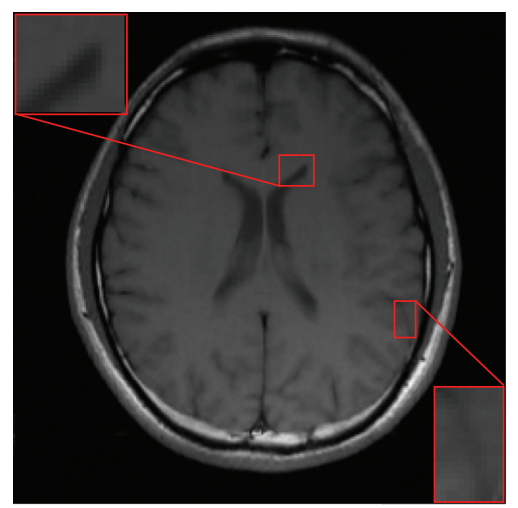

(d)

FIgURE 6: (a) Reference. Reconstructed images from the radial sampling mask with accelerating factor $R=4$ by (b) CSMRI-TV, (c) DLMRI, and (d) our proposed DDTF-MRI, respectively.

on fixed transform, it is the fastest. However, this efficiency comes at the cost of accuracy. DLMRI takes the longest time in all cases. On the other hand, DDTF-MRI has achieved the best reconstruction result without taking the longest reconstruction time, which is due to three aspects: (1) $\ell_{1}$ norm is used in DDTF-MRI instead of $\ell_{0}$ norm. While $\ell_{0}$ norm minimization is a NP-hard problem, $\ell_{1}$ norm minimization is convex and can be solved with much less time while promoting sparsity; (2) the tight frame used in this paper ensures that the given signal can be perfectly represented by its canonical expansion as $W^{T} W=I$; therefore it can be efficiently implemented; (3) two-level Bregman iteration technique is used in the proposed method, which can further promote fast and accurate MR image reconstruction. So the proposed method has improved the accuracy without sacrificing too much efficiency.

\section{Conclusions}

A DDTF-MRI method has been proposed in this paper to effectively reconstruct MR image from undersampled $K$-space data. DDTF-MRI trains an adaptive tight frame for each to-be-reconstructed image. Furthermore, a twolevel Bregman iteration algorithm has been developed to solve the proposed model. Results on both simulated and in vivo datasets demonstrate the superior performance of
DDTF-MRI over the other two state-of-the-art MR image reconstruction methods, including CSMRI-TV and DLMRI, in artifacts suppression and edge preservation. Although DDTF-MRI is slower than the fixed transform based method CSMRI-TV, its accuracy is much higher than CSMRI-TV. Furthermore, as an adaptive training method, DDTF-MRI is much faster than the typical training method, DLMRI. This indicates that our method can improve the reconstruction accuracy without introducing too much computation load. In the future, we may further optimize the implementation and consider sparser representations.

\section{Conflict of Interests}

The authors declare that there is no conflict of interests regarding the publication of this paper.

\section{Authors' Contribution}

Jianbo Liu and Shanshan Wang contributed equally to this work.

\section{Acknowledgments}

The authors sincerely thank the anonymous referees for their valuable comments on this work. The authors would 
also like to thank Lustig and Ravishankar for sharing their experiment materials and source codes. This work was partly supported by China NSFC 61102043, 11301508, 81120108012, 61471350, and KQCX20120816155710259 and Shenzhen Basical Research Project (JCYJ20140610152828678).

\section{References}

[1] Z. P. Liang and P. C. Lauterbur, Principles of Magnetic Resonance Imaging: A Signal Processing Perspective, Wiley-IEEE Press, 2000.

[2] M. Lustig, D. Donoho, and J. M. Pauly, "Sparse MRI: the application of compressed sensing for rapid MR imaging," Magnetic Resonance in Medicine, vol. 58, no. 6, pp. 1182-1195, 2007.

[3] E. J. Candès, J. Romberg, and T. Tao, "Robust uncertainty principles: exact signal reconstruction from highly incomplete frequency information," IEEE Transactions on Information Theory, vol. 52, no. 2, pp. 489-509, 2006.

[4] D. L. Donoho, "Compressed sensing," IEEE Transactions on Information Theory, vol. 52, no. 4, pp. 1289-1306, 2006.

[5] S. Ravishankar and Y. Bresler, "MR image reconstruction from highly undersampled k-space data by dictionary learning," IEEE Transactions on Medical Imaging, vol. 30, no. 5, pp. 1028-1041, 2011.

[6] Q. Liu, S. Wang, K. Yang, J. Luo, Y. Zhu, and D. Liang, "Highly Undersampled magnetic resonance image reconstruction using two-level Bregman method with dictionary updating," IEEE Transactions on Medical Imaging, vol. 32, no. 7, pp. 1290-1301, 2013.

[7] Z. Shen, "Wavelet frames and image restorations," in Proceedings of the International Congress of Mathematicians, vol. 4, pp. 28342863, 2010.

[8] E. J. Candès, "Ridgelets: estimating with ridge functions," The Annals of Statistics, vol. 31, no. 5, pp. 1561-1599, 2003.

[9] E. J. Candès and D. L. Donoho, "New tight frames of curvelets and optimal representations of objects with piecewise $c^{2}$ singularities," Communications on Pure and Applied Mathematics, vol. 57, no. 2, pp. 219-266, 2004.

[10] E. Le Pennec and S. Mallat, "Sparse geometric image representations with bandelets," IEEE Transactions on Image Processing, vol. 14, no. 4, pp. 423-438, 2005.

[11] J.-F. Cai, R. H. Chan, and Z. Shen, "A framelet-based image inpainting algorithm," Applied and Computational Harmonic Analysis, vol. 24, no. 2, pp. 131-149, 2008.

[12] I. Daubechies, B. Han, A. Ron, and Z. Shen, "Framelets: MRAbased constructions of wavelet frames," Applied and Computational Harmonic Analysis, vol. 14, no. 1, pp. 1-46, 2003.

[13] R. H. Chan, S. D. Riemenschneider, L. Shen, and Z. Shen, “Tight frame: an efficient way for high-resolution image reconstruction," Applied and Computational Harmonic Analysis, vol. 17, no. 1, pp. 91-115, 2004.

[14] Y. Hu and M. Jacob, "Higher degree total variation (HDTV) regularization for image recovery," IEEE Transactions on Image Processing, vol. 21, no. 5, pp. 2559-2571, 2012.

[15] D. Liang, H. Wang, Y. Chang, and L. Ying, "Sensitivity encoding reconstruction with nonlocal total variation regularization," Magnetic Resonance in Medicine, vol. 65, no. 5, pp. 1384-1392, 2011.
[16] G. Kutyniok and D. Labate, "Construction of regular and irregular shearlet frames," Journal of Wavelet Theory and Applications, vol. 1, no. 1, pp. 1-12, 2007.

[17] M. Akcakaya, S. Nam, P. Hu et al., "Compressed sensing with wavelet domain dependencies for coronary MRI: a retrospective study," IEEE Transactions on Medical Imaging, vol. 30, no. 5, pp. 1090-1099, 2011.

[18] X. Qu, D. Guo, B. Ning et al., "Undersampled MRI reconstruction with patch-based directional wavelets," Magnetic Resonance Imaging, vol. 30, no. 7, pp. 964-977, 2012.

[19] Y. Liu, J. F. Cai, Z. Zhan et al., "Balanced sparse model for tight frames in compressed sensing magnetic resonance imaging," PLOS ONE, vol. 10, no. 4, Article ID e0119584, 2015.

[20] J.-F. Cai, H. Ji, Z. Shen, and G.-B. Ye, "Data-driven tight frame construction and image denoising," Applied and Computational Harmonic Analysis, vol. 37, no. 1, pp. 89-105, 2014.

[21] M. Elad and M. Aharon, "Image denoising via sparse and redundant representations over learned dictionaries," IEEE Transactions on Image Processing, vol. 15, no. 12, pp. 3736-3745, 2006.

[22] Q. Liu, S. Wang, L. Ying, X. Peng, Y. Zhu, and D. Liang, "Adaptive dictionary learning in sparse gradient domain for image recovery," IEEE Transactions on Image Processing, vol. 22, no. 12, pp. 4652-4663, 2013.

[23] Q. Wang, J. Liu, N. Janardhanan, M. Zenge, E. Mueller, and M. S. Nadar, "Tight frame learning for cardiovascular MRI," in Proceedings of the IEEE 10th International Symposium on Biomedical Imaging: From Nano to Macro (ISBI '13), pp. 290293, IEEE, April 2013.

[24] J.-F. Cai and Z. Shen, "Framelet based deconvolution," Journal of Computational Mathematics, vol. 28, no. 3, pp. 289-308, 2010.

[25] S. Osher, M. Burger, D. Goldfarb, J. Xu, and W. Yin, "An iterative regularization method for total variation-based image restoration," Multiscale Modeling \& Simulation, vol. 4, no. 2, pp. 460-489, 2005.

[26] J.-F. Cai, S. Osher, and Z. Shen, "Split bregman methods and frame based image restoration," Multiscale Modeling and Simulation, vol. 8, no. 2, pp. 337-369, 2009.

[27] W. Yin, S. Osher, D. Goldfarb, and J. Darbon, "Bregman iterative algorithms for $l_{1}$-minimization with applications to compressed sensing," SIAM Journal on Imaging Sciences, vol. 1, no. 1, pp. 143$168,2008$.

[28] D. O. Walsh, A. F. Gmitro, and M. W. Marcellin, "Adaptive reconstruction of phased array MR imagery," Magnetic Resonance in Medicine, vol. 43, no. 5, pp. 682-690, 2000.

[29] Z. Wang, A. C. Bovik, H. R. Sheikh, and E. P. Simoncelli, "Image quality assessment: from error visibility to structural similarity," IEEE Transactions on Image Processing, vol. 13, no. 4, pp. 600$612,2004$. 


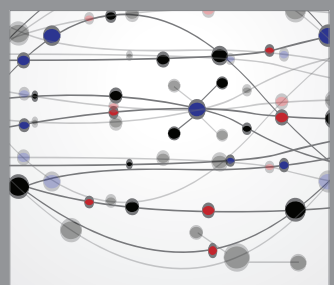

The Scientific World Journal
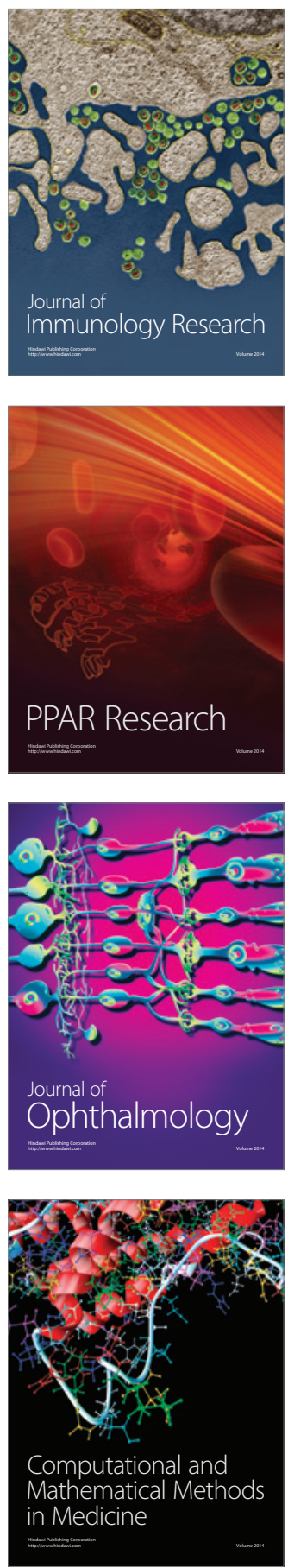

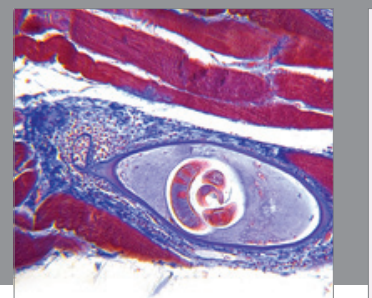

Gastroenterology

Research and Practice
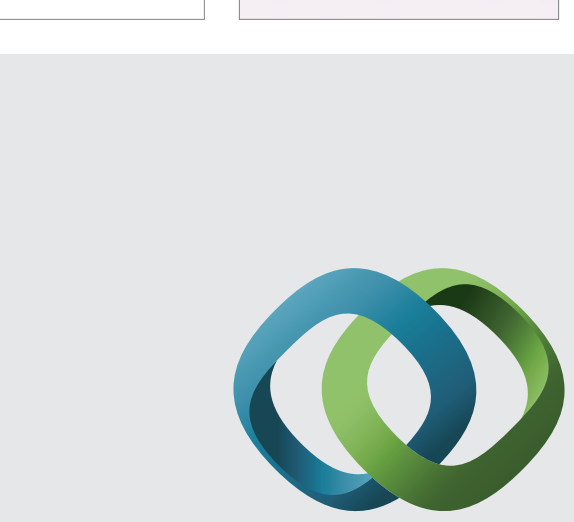

\section{Hindawi}

Submit your manuscripts at

http://www.hindawi.com
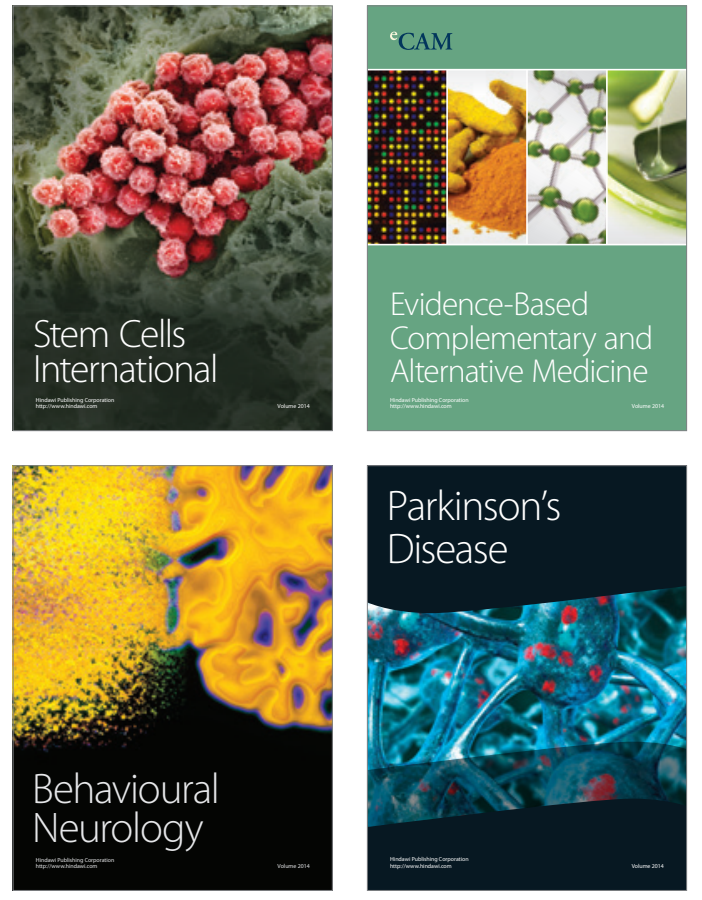
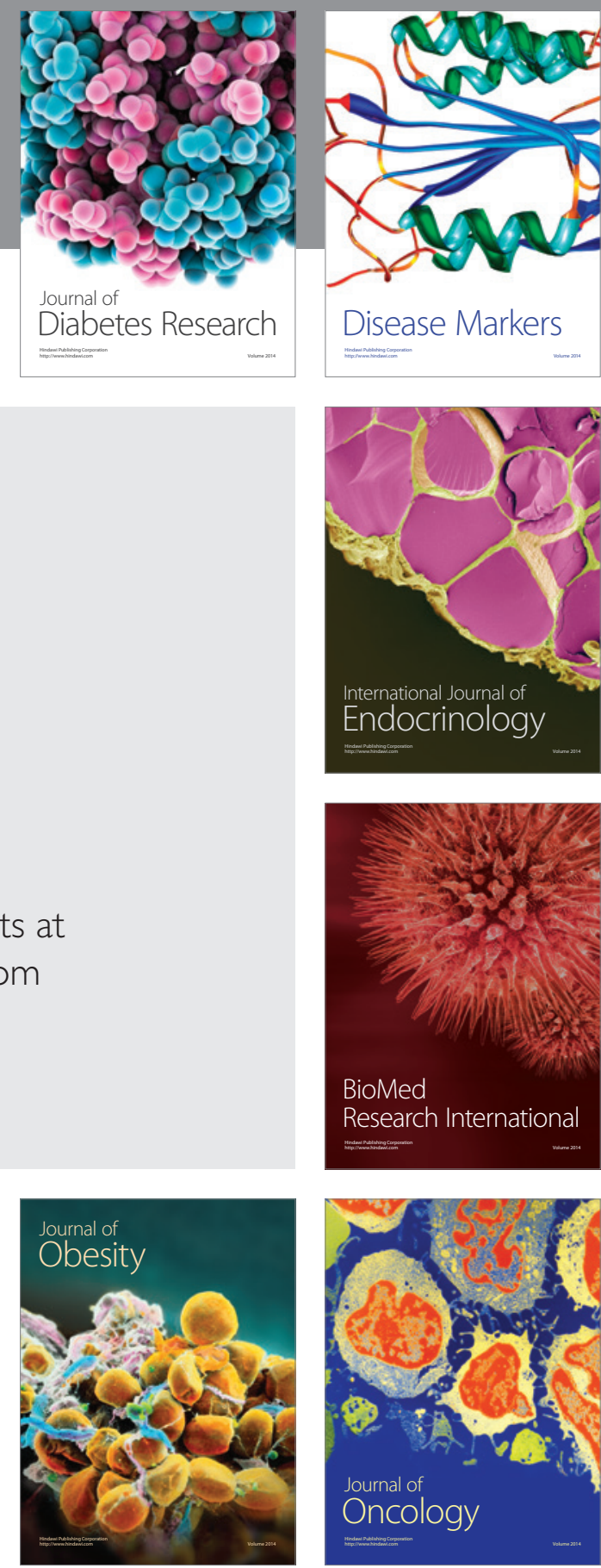

Disease Markers
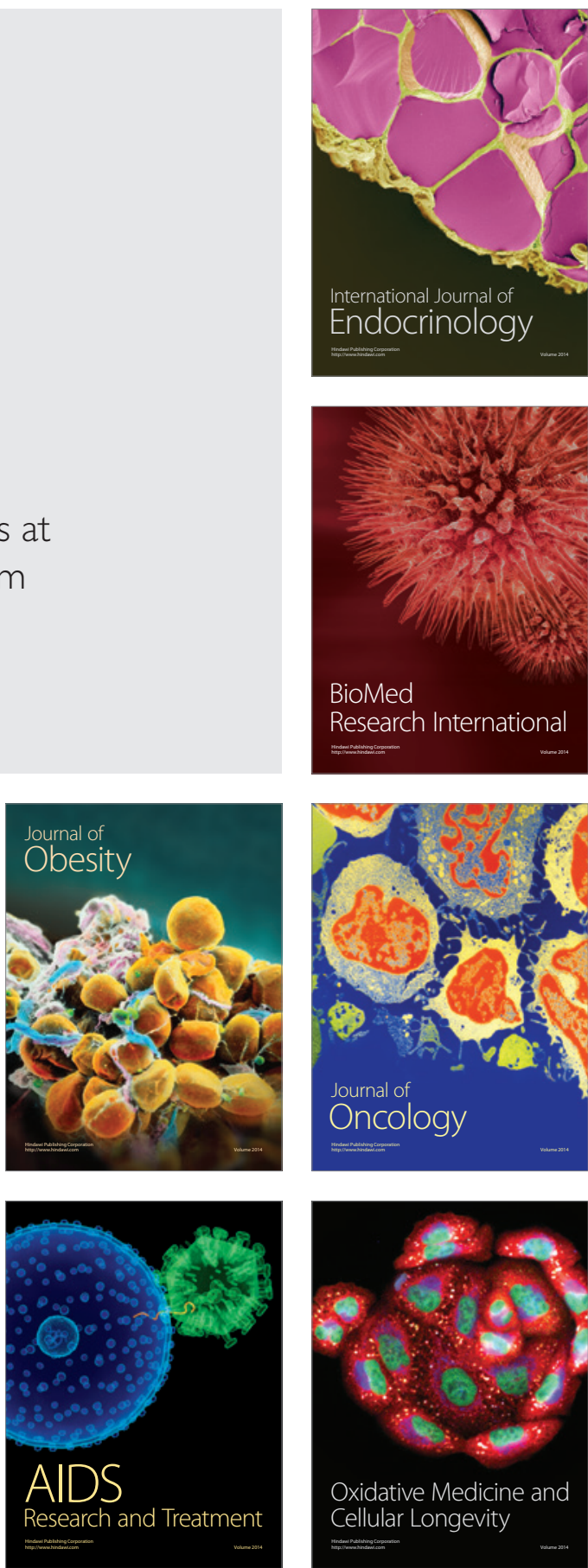